\title{
Una rara causa de paro cardiaco
}

\section{A rare cause of cardiac arrest}

\author{
Germán Devia, Emiro Valverde, Libardo Marmolejo
}

\begin{abstract}
Resumen
La parada cardiaca en su mayoría es secundaria a enfermedad coronaria, este artículo hace referencia a un paciente con una paro cardiaco secundario a un infarto agudo del miocardio con elevación del ST que se estudió documentando arterias epicárdicas sanas, pero con la presencia de una fístula entre la arteria descendente anterior hacia una lesión neoplásica de mediastino que previamente se había documentado en estudios imagenológicos anteriores. Las fístulas coronarias son raras en su presentación, este caso demuestra una fístula asociada a la presencia de un tumor del mediastino que se presenta al servicio de urgencias en parada cardiaca, una causa bizarra en la determinación de la etiología del paro. (Acta Med Colomb 2016; 41: 71-73).
\end{abstract}

Palabras clave: paro cardiaco, fístula coronaria, infarto con elevación del ST.

\begin{abstract}
Cardiac arrest is mostly secondary to coronary disease; this article refers to a patient with cardiac arrest secondary to acute myocardial infarction with ST elevation who was studied documenting healthy epicardial arteries, but with the presence of a fistula between the left anterior descending artery into a neoplastic lesion of the mediastinum that was documented in previous imaging studies. Coronary fistulae are rare in its presentation; this case demonstrates a fistula associated with the presence of a tumor of the mediastinum of a patient admitted in the emergency department in cardiac arrest being a bizarre cause in determining the etiology of cardiac arrest. (Acta Med Colomb 2016; 41: 71-73).

Keywords: cardiac arrest, coronary fistula, infarction with ST elevation.
\end{abstract}

Dr. Germán Devia: Especialista en Medicina de Emergencias, Hospital Universitario Mayor, Instructor de Práctica Departamento de Medicina de Emergencias, Universidad del Rosario; Dr. Emiro Valverde: Residente Tercer Año de Medicina de Emergencias, Universidad del Rosario; Dr. Libardo Marmolejo: Médico General, Universidad del Rosario, Hospital Universitario Mayor. Bogotá, D.C. (Colombia).

Correspondencia: Dr. Germán Devia. Bogotá, D.C. (Colombia)

E-mail: germandevia@gmail.com

Recibido: 16/IX/2015 Aceptado: 23/II/2016

\section{Introducción}

Una fístula arterial coronaria es una conexión anormal que vincula directamente una o más arterias coronarias a una cámara del corazón o a los principales vasos torácicos sin un lecho capilar interpuesto (1), se pueden clasificar en fístulas coronariocamerales, mientras que las que terminan en una vena se pueden llamar fístulas coronarias arteriovenosas (2), estas lesiones pueden ser observadas entre 0.05-0.25\% de todos los estudios coronarios (3), en este artículo nosotros describimos un caso de una parada cardiaca secundaria a un evento coronario agudo que se estudió y se consideró secundario a una inusual forma de presentación de una fístula arterial coronaria.

\section{Presentación del caso}

Se trata de un paciente masculino de 45 años quien se encontraba hospitalizado por el servicio de oncología remitido de otra institución fuera de la ciudad por cuadro de dolor en tórax retroesternal, no irradiado, asociado a tos, con expectoración blanca y fiebre no cuantificada, adicional a la sintomatología manifestaba disnea progresiva. Fue estudiado extrahospitalariamente y por encontrar leucocitosis severa, con imagen que interpretaron inicialmente como sugestiva de neumonía, deciden dar manejo antibiótico con poca respuesta.

Hacen una tomografía como parte de su estudio que documenta una gran masa del mediastino, sugestiva de linfoma y envían a nuestra institución para manejo médico. En la estancia el paciente fue valorado por oncología quienes documentan un tumor germinal tipo seminoma extragonadal, le hacen una nueva tomografía que documenta una masa del mediastino anterior, con infiltración a pericardio, contacto con la arteria pulmonar y con la vena que drena el lóbulo superior izquierdo. Sin lesiones neoplásicas adicionales en estudios de cuello y abdomen. Realizan ecocardiograma que muestra contractilidad ventricular izquierda normal fracción de eyección de $49 \%$ con derrame pericárdico moderado a severo posterior sin signos de taponamiento, sin masas ni trombos en el estudio. Se caracterizó el tumor con biopsia y se diagnosticó un tumor maligno pobremente diferenciado 
germinal tipo seminoma con inmunohistoquímica cd117 (+), ACL B y T, CAAE1/AE3, CK7 y CK20, TIF1, cromogranina (-). Se le propuso manejo con quimioterapia con intención curativa protocolo PEB de manera prioritaria.

Al tercer día el paciente presenta de manera súbita pérdida del estado de conciencia en sala de quimioterapia, le documentan ausencia de pulso y activan protocolo de resucitación de la institución por lo que el servicio de emergencias acude a la sala de oncología. Se encuentra a un paciente con esfuerzo respiratorio agónico, monitorizado y con la presencia de taquicardia ventricular que progresa a asistolia, se procede a intubación en secuencia crash sin complicaciones, se realizan maniobras de resucitación por aproximadamente 20 minutos y se logra retorno a circulación espontánea, se documenta en ultrasonido a la cabecera del paciente derrame pericárdico sin evidencia de taponamiento, con pobre contractilidad, se descarta hemo o neumotórax, las cavidades cardiacas se encontraron de tamaños usuales, se realiza electrocardiograma (EKG) el cual documenta lesión subepicárdicas de la cara anterior, la cual no era conocida en EKG previo (Figura 1), se le da aviso a grupo de hemodinamia y se traslada a sala de procedimientos angiográficos donde realizan arteriografía coronaria de emergencia, que reporta coronarias epicárdicas sin evidencia de placas arterioescleroticas; sin embargo, en la arteria descendente anterior reportan que "se observan ramas anteriores hacia una estructura que no se logra identificar en el aspecto más anterior, superior y proximal de la arteria y que podría corresponder a pequeñas fístulas a un hemangioma o irrigación de estructura neoplásica infiltrante" (Figura 2), se hace ecocardiograma posparo que documenta deterioro de la eyección a $35 \%$ con aquinesia anterior tercio distal y con imagen compatible con trombo apical, con derrame pericárdico moderado sin evidencia de taponamiento cardiaco. Así mismo se obtuvo una radiografía de tórax pos paro donde es evidente la masa mediastinal (Figura 3).

El paciente fue trasladado a la unidad de cuidado intensivo (UCI) donde requirió de soporte ventilatorio y vasopresor por 48 horas y luego de 72 horas en la UCI es dado de alta a piso donde continuó con el plan de manejo oncológico del tumor. Se consideró que el paciente presentó una parada cardiaca secundaria a infarto agudo del miocardio tipo II por fenómeno secundario a fístula coronaria para la irrigación a tumor mediastinal.

\section{Discusión}

Para la clasificación de las fístulas se encuentran las que drenan a cavidades cardiacas, o a estructuras vasculares venosas. Considerando el caso expuesto, esta clasificación no incluye fenómenos vasculares hacia tumores del mediastino, dado lo raro de estas fístulas, en la literatura no existen reportes de casos similares al descrito. No es compatible el caso con un fenómeno de robo coronario debido a que el sistema coronario se encontraba sin enfermedad obstructiva, siendo esta condición requerida para que se de este fenómeno (4), en el caso del paciente el sistema coronario era sano y no existían anomalías anatómicas vasculares en el árbol coronario.

Las fístulas coronarias ocurren igual en ambos sexos con una incidencia de $0.003 \%$ en la población general (5), lo que hace que este tipo de lesiones se diagnostique de manera poco frecuente y no se diagnostican de manera tardía o incluso incidental. Las fístulas adquiridas son el resultado de lesiones iatrogénicas como posoperatorios cardiovasculares, implantes de dispositivos vasculares, biopsias transbronquiales; o incluso traumáticas que pueden ser secundarias a lesiones traumáticas penetrantes, o cerradas (6).

De acuerdo con la anatomía coronaria, de $50-60 \%$ son originadas de la arteria coronaria derecha, $30 \%$ de la descendente anterior y $15 \%$ de la circunfleja; cerca de $90 \%$ de las fístulas que son coronariocamerales drenan a las cámaras cardiacas derechas (7), en especial el ventrículo derecho en $40 \%$ (7). En general las fístulas congénitas son asintomáticas hasta los 18 años y se pueden manifestar por disnea con el ejercicio (8), arritmias auriculares, hipertensión pulmonar, insuficiencia cardiaca, disnea paroxística nocturna e incluso angina, en especial si el paciente tiene un contexto de enfermedad arterioesclerótica de base (6).

La presentación clínica del paciente puede variar de acuerdo con el tamaño de la fístula, la edad del paciente y la presencia de isquemia miocárdica (9). Un infarto agudo del miocardio puede ocurrir secundario a la disminución del flujo coronario distal a la fístula aun si el paciente se

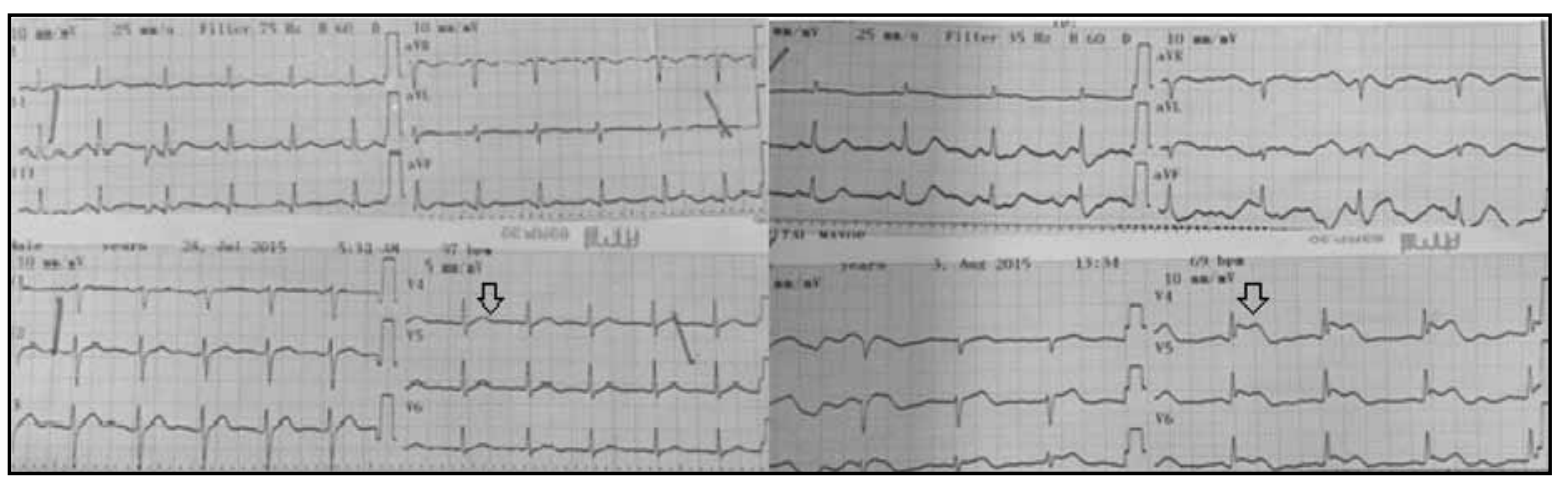

Figura 1. A. EKG de base del paciente, B. Cambios eléctricos de la cara anterior, posparo. 


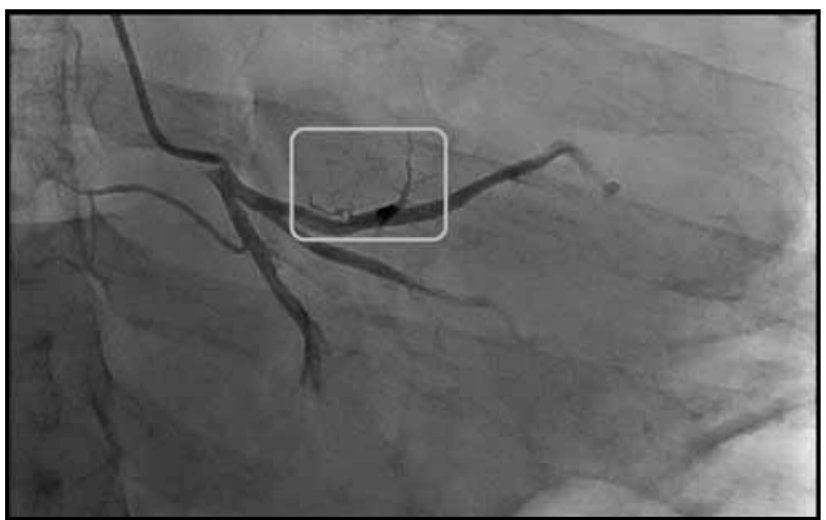

Figura 2. Arteriografía coronaria con ramas de la arteria descendente anterior hacia la lesión neoplásica

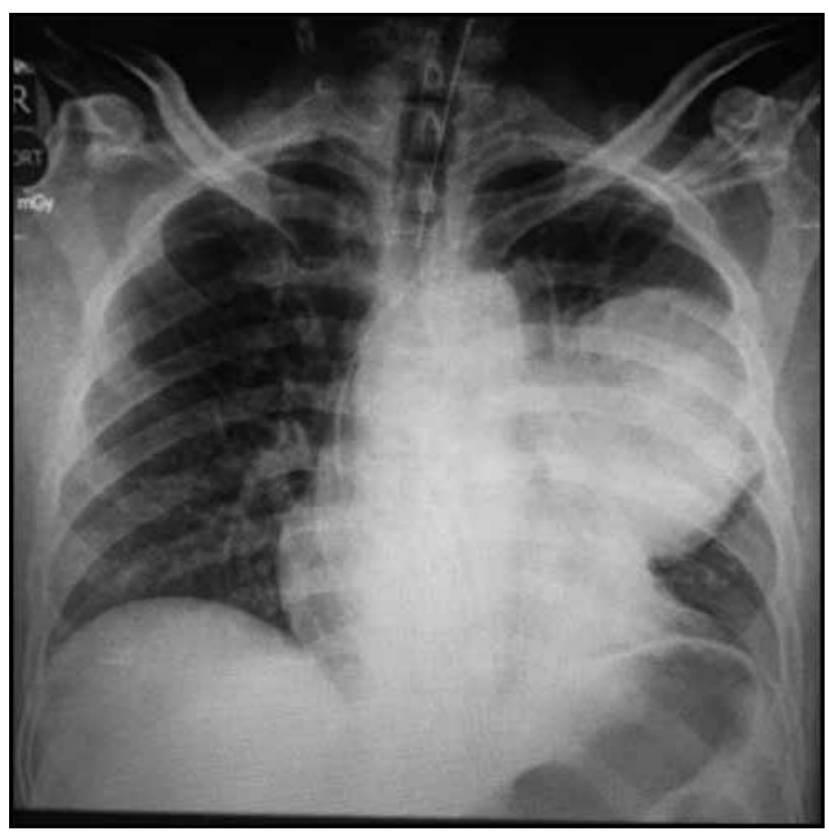

Figura 3. Radiografía pos parada cardiaca del paciente en donde es evidente la lesión tumoral mediastinal.

encuentra sin evidencia de arterioesclerosis (6) como es el caso del paciente reportado en este escrito. Ocasionalmente puede presentarse como un derrame pericárdico o incluso una muerte súbita $(10,11)$. La historia natural de las fístulas es variable pueden llegar a trombosarse espontáneamente (12), pero se recomienda la terapia antiagregante como parte del manejo, en especial en fístulas distales y con anormalidades en las arteriales coronarias $(6,10)$. La angina puede ser tratada con medidas usuales como betabloqueadores, calcioantagonistas o nitratos (13).

La principal indicación para el cierre son la falla cardiaca y los síntomas de isquemia miocárdica (14); sin embargo, estas indicaciones siguen siendo tema de debate. En general el pronóstico luego del cierre quirúrgico es bueno, pero exis- te la posibilidad de recanalización, dilatación de la arteria coronaria y ostium, formación de trombos calcificación e incluso isquemia miocárdica y por esta razón se debe hacer seguimiento periódico del paciente (15), los pacientes que no son operados pueden pasar tiempos prolongados asintomáticos (16).

\section{Conclusión}

Las fístulas coronarias son raras y de presentación tardía en su mayoría, para el médico de emergencias se deben sospechar en pacientes con clínica de falla cardiaca sin causa evidente o en el contexto de una angina y hasta en casos de infarto agudo del miocardio con o sin elevación del segmento ST, el tratamiento cuando las fístulas son sintomáticas es en principio quirúrgico, sin embargo aún existe debate al respecto. El manejo médico incluye el manejo usual para enfermedad coronaria con ácido acetilsalicílico y betabloqueadores.

\section{Financiación}

Financiado con recursos propios de los autores, los autores declaran no tener conflictos de interés en relación con la presentación de este manuscrito.

\section{Referencias}

1. Ashraf SS, Shaukat N, Fisher M, Clarke B, Keenan DJ. Bicoronary-pulmonary fistulae with coexistent mitral valve prolapse: a case report and literature review of coronary-pulmonary fistula. Eur Heart J. 1994;15: 571-4.

2. Tiryakioglu S, Gocer H, Tiryakioglu O, Kumbay E. Multiple coronary-cameral fistulae.Tex Heart Inst J. 2010; 37: 378-9.

3. Kamineni R, Butman SM, Rockow JP, Zamora R. An unusual case of an accessory coronary artery to pulmonary artery fistula: successful closure with transcatheter coil embolization. J Interv Cardiol. 2004; 17: 59-63.

4. Zimarino M, D'Andreamatteo M, Waksman R, Epstein SE, De Caterina R. The dynamics of the coronary collateral circulation. Nat Rev Cardiol. 2014; 4: 191-7.

5. Luo L, Kebede S, Wu S, Stouffer GA. Coronary artery fistulae. Am J Med Sci. 2006; 332: 79-84.

6. Loukas M, Germain AS, Gabriel A, John A, Tubbs RS, Spicer D. Coronary artery fistula: a review. Cardiovasc Pathol. 2015; 24 (3): 141-8.

7. Qureshi S. Coronary arterial fistulas. Orphanet J Rare Dis. 2006; 1: 51.

8. Rittenhouse EA, Doty DB, Ehrenhaft JL. Congenital coronary artery-cardiac chamber fistula: review of operative management. Ann Thorac Surg. 1975; 20: 468-85.

9. Gowda RM, Vasavada BC, Khan IA. Coronary artery fistulas: clinical and therapeutic considerations. Int J Cardiol. 2006; 107 (1): 7-10.

10. Lau G. Sudden death arising from a congenital coronary artery fistula. Forensic Sci Int. 1995; 73: 125-30.

11. Ozeki S, Utsunomiya T, Kishi T, et al. Coronary arteriovenous fistula presenting as chronic pericardial effusion. Circ J. 2002; 66: $779-82$.

12.Sapin P, Frantz E, Jain A, Nichols TC, Dehmer GJ. Coronary artery fistula: an abnormality affecting all age groups. Medicine. 1990; 69: 101-3.

13. Mechleb W, Abboud L, Mattar C, Haddadin T. Case report and review of literature: coronary artery fistulae. Tenn Med. 2013; 106: 39-41.

14. Balanescu S, Sangiorgi G, Castelvecchio S, Medda M, Inglese L. Coronary artery fistulas: clinical consequences and methods of losure: a literature review. Ital Heart J. 2001; 2: 669 - 76.

15. Pettersen MD, Ammash NM, Hagler DJ, Rihal CS, Cabalka AK. Endovascular stent implantation in a coronary artery to pulmonary artery fistula in a patient with pulmonary atresia with ventricular septal defect and severe cyanosis. Cath Cardiovasc Interv. 2001; 54: 358 - 62.

16. Said SA, Landman GH. Coronary - pulmonary fistula: long-term follow-up in operated and non-operated patients. Int J Cardiol. 1990; 27: 203 - 10. 